suffered from enlargement of the gland for many years, and various plans had been adopted for removal of the gland or for reduction of its size. A short time before she came under our care the tumour had been pierced by the needle, and the electric cautery had been brought into requisition several times. At the last of these attempts the needle had broken, and from three to four inches of it remained permanently in the gland. This was sometimes a cause of pain, but was quite supportable, and was probably no more than an occasional inconvenience. The serious part of the case was that the growth continued, that the skin was distended to the extremest degree, that the trachea was pushed far on one side, that the venous channels through the neck were obstructed, and that the oesophagus was so constricted from the pressure that nothing but fluid food could be swallowed, and even that with difficulty.

In this condition we endeavoured to remove the gland section by section by the subcutaneons destruction of it from injection of the ethylate. Selecting the part where pressure from within was most declared we injected thirty minims of the solution into the structure of the gland at the distance of about an inch from the surface. The operation was followed by some slight local heat and throbbing, which passed away in a few hours, after which there was a very distinct reduction of size and tension of the gland at the part injected. In a few days we injected again with similar good results, and after six injections the gland was reduced to at least a third of its size, with the greatest relief to the breathing and complete relief to the cesophagus. We were congratulating ourselves on our success when an accidental check succeeded. After coming to my house to have a further subcutaneous injection the patient, who insisted on walking home, was immersed suddenly in a dense London fog with rain. Losing her way for a time, she reached her home at last wet through, extremely exhausted and suffering from cold. She was in this way subjected to an acute attack of bronchitis, with severe cough and expectoration, in the course of which the thyroid became congested and inflamed. Abscess formed in the gland, followed by free discharge of pus. In spite of all she, nevertheless, began to recover and to get up and attend for some weeks to a part of her daily duties, but with an im pression fixed on her mind that she should die, and a constant expression that she wished to die. One evening, after making preparation for Christmas festivities, she summoned all the servants under her control, gave some of them presents, expressed regret that she had chided one of them, and then bade them all good-by and dismissed them from her room. A little later a loud ringing brought them back, to find her on the floor with the wound in the neck open and bleeding freely. Dr. Sedgwick was quickly in attendance, and found that she was dead from the loss of blood from one of the large veins involved in the gland. Whether bleeding from the wound alarmed her and led her to remove the dressings, or whether she ruptured the vein herself by interfering with the dressings, we could not tell, but the accidental mode of termination does not to my mind affect the treatment at all in regard to principle; and should another instance of enlarged thyroid come before me in which death from pressure on the cesophagus is inevitable, I shall not for a moment hesitate to suggest the same mode of destroying the gland in sections by the subcutaneous injection of the ethvlate.

There are some minor uses for the ethylate, which are not without their practical value. I have used it successfully for removing warts, for destroying a small melanotic growt on the face, for a ringworm which had long withstood other forms of local treatment, for the removal of a small loose hæmorrboid, and for the destruction of the vascular growth dependent on an ingrowing nail. In fact, the ethylate becomes after a little practice with it like a kind of supple. mentary knife, and in many cases takes the place of the knife without surgesting the fears that are so of ten connected with that useful but dreaded instrument.

One or two words as to the mode of application. In my first paper I recommended the exclusive use of a glass rod for applying the solution. As I have become more familiar with the treatment I have more commonly used a brush of camel's hair, as more readily applicable. The only objection to the brush is that one using of it destroys it-a trifling objection. Occasionally, when I wish to put on the solution in fine points or lines, I employ a new quill pen, taking the solution up with it in the same way as ink would be taken if the pen were about to be applied for its usual purpose.
In this manner the fluid can be applied drop by drop or in line to the part, and its action can be confined precisely to the spot required.

When the solution is used in the form of spray, it should be diluted with absolute alcohol, sp. gr. 795, and sprayed through a Seigle's disperser. The operator in using the spray must be careful not to inhale it himself. In spraying it one day, a little of the fine spray was reflected back into my face, and $I$ inhaled a breath of it, the effect of which was to cause a soreness and irritation of the throat, which lasted some hours. This, however, need not cause alarm. A weak solution can be actually sprayed into the throat, and in cases of malignant sore-throat might prove of great service.

To relieve the pain that is connected with the application of the ethylate, an alcoholic solution of opium may be added with good effect, but for this purpose chloroform must never be used. Chloroform mixed with the ethylate may explode with some violence. All the alcohols treated with sodium or potassium yield a sodium or potassium substitute, and I have made an amylate, a butyrate, and other similar compounds. Whether they will prove useful in medicine remains to be seen. Again, whether potassium ethylate has any advantages over sodium ethylate remains also to be seen. I may relate of potassium ethylate that it is much keener in its action than its sodium ally. It is nearer to the knife, and might almost, rival the knife when used in its concentrated form. I have no doubt it will one day come into extensive application, for the whole study of these pectising alcoholic caustics is but in its infancy. For my part, I am repaid already in having been the pioneer in the study, and whatever successes my more learned successors may achieve from it, I most heartily oller them welcome and good-will.

\section{EPITOME OF A PAPER ON THE NATURE AND RELATIONS OF THE DISEASES OF THE EAR.}

BY ROBERT SINCLAIR, M.D.,

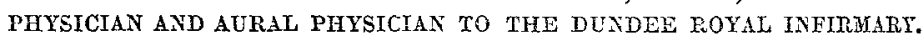
(Concluded from p.208.)

THE symptoms common to all acute inflammations are familiarly known by the description of Celsus, "rubor $e t$ tumor, cum calore et dolore." The symptoms which, in addition to these, more specially characterise the disease under consideration, are impairment of hearing, giddiness, a sense of fulness in the head, and increase of pain on mastication, swallowing, moving the head, and blowing the nose. If the ear be inspected in the earliest stage of the attack by means of a speculum and a mirror of $5^{\prime \prime}$ or $6^{\prime \prime}$ focal distance, signs of vascular injection will be seen in the part first affected, which will be the dermoid layer of the membrana tympani, if the disease has originated in the external meatus, and the lining membrane of the tympanum and the mucous layer of the membrana tympani if it has taken its origin from the usual source, the pharynx. Should the attack be a very slight one or be appropriately treated at first, resolution occurs, and in a short time the illness terminates without leaving any evil results behind. But if left to itself in the milder cases effusion of mucus occurs, and in the more severe suppuration. These processes usually occur within forty-eight hours of inception, a fact which gives us a very significant hint for early and rational treatment. During this stage there is usually bulging of the drum-head, observable on inspection, and all the lscal and general symptoms become much more severe. It is even yet possible for the patient to be relieved withont surcical aid by the escape of the effusion down the Eustachian tube. But as this channel is ustally swollen and blocked up, such a happy result is exceedingly rure. The more usual course for the effusion to follow is the one we would natu. rally expect-namely, the direction of the point of least resistance-the membrana tympani, aud the patient is fortunate indeed if rupture occur before serious disorganisation of the organ takes place. But his troubles are by no means at an end if his medical adviser is sceptical of the propriety of meddling with such a "useful safety valve" as a "trifling 
discharge from the ear." He has before him the possibilities of a life-long disgusting suppuration and irremediable deafness. If he be a child who has not learned, or has only recently acquired articulate speech, deaf-mutism is almost a certainty. And in any case he may at any moment fall a victim to meningitis, cerebral abscess, or pyæmia.

To say that the only foundation for the rational and suecessful treatment of this disease is to be found in the general principles which guide us in the treatment of inflammation is, I am aware, to utter a most obvions truism. But it is, nevertheless, a truism which has too largely shared the neglect proverbially attached to things familiar.

In the first place, we must, as far as possible, remove the cause, and prevent the operation of other possible causes. These objects we attain by attending to the external meatus and the pharynx, and in children by lancing swollen gums.

Secondly, we must procure absolute rest for the organ, and, as far as possible, physiological repose for the body generally. With this aim we place the patient in bed, in a well-ventilated and otherwise comfortable apartment; procure for him the greatest possible quietness by diminishing talking and noises to a minimum, and by placing a pledget of absorbent cotton wadding in the ear; and permit no fuod requiring mastication, for mastication obviously interferes with rest of the organ.

Thirdly, we must moderate the local action by general and local measures. To accomplish this end we must, in the first place, attend to the state of the excretory organs. A judicious selection of vascular sedatives and narcoticssuch as aconite, tartar emetic, opium, and chloral-inust be made, according to the requirements of each case. And, above all, there should be no hesitation in adopting the aid of four trusty ally, bloodletting by means of leeches. As the situation selected for this measure is a matter of prime importance, I shall ask your attention for a moment to the vascular supply of the organ. The posterior auricular, the anterior auricular branches of the temporal, and the deep auricular branch of the internal maxillary are the arteries of the external meatus. The last-named vessel is the souree of the principal arterial supply of the membrana tympani. The stylo-mastoid branch of the posterior auricular reaches the tympanum through the arueduct of Fallopius, and the tympanic branch of the internal maxillary enters by the fissure of Glasser. These are the two chief arteries of the tympanum.

From these facts it would appear that leeches should be applied in front of and below the external meatus, and certainly not over the mastoid, unless there is evidence that the mastoid cells are also implicated, in which case the parts both before and behind the meatus should be bled. In a child two or three, and in the adult not less than six, leeches should be applied. The meatus should be carefully plugged with wadding before the operation, to prevent the leeches and blood entering it. After the operation the bites should be carefully covered with plaster, to prevent their inoculation with pus, and fomentations by sponges wrung out of hot water, or, what is better, by warm water syringing of the meatus, should be sedulously adopted. "Never poultice an eye or an ear" is an injunction that cannot be too often repeated, for in neither organ can we afford to rum the risk of suppuration and its consequences.

Lastly, if the products of inflammation have accumulated, it becomes our business to procure for them a safe and speedy exit by means of the knife. For the performance of this operation we require good light, a wide speculum, a concave mirror of $5^{\prime \prime}$ or $6^{\prime \prime}$ focal distance, attached by band to the forehead, and a delicate, sharp-pointed knife. Many instruments have been devised for making the incision. But the one which seems to me to falfil all the requirements in the highest degree is the myringotome by Dr. Woakes. This beautiful instrument has the supreme merit of perfect safety. It is a sharp-pointed, double-bladed knife, con cealed in a cannula, worked by pressure on a spring, and fixed in an angularly-placed handle. In accordance with a well-known principle of surgery, a free incision should be made at the bulging point, if one can be clearly detected; and if not, in the lower seoment of the membrane, behind o in front of the handle of the malleus. A good deal can be said for both positions. After the fluid has been evacuated, the inflamed surface should be washed every few hours by copious injections of water about the temperature of the blood, with the double view of diminishing inflammatory action and removing its products. The best way to do this is by means of a brass syringe, fitted with a somewhat conical-shaped nozzle to fit into the meatus, and ensure the thorough washing of the meatus, tympanic cavity, and Eustachian tube. To use a small glass or pewter syringe is merely to trifle with a serious inflammation and the fate of an important organ. A well-made Higginson's or indiarubber ball syringe fitted with a proper nozzle is, however, a good substitute for the brass syringe. In the intervals of syringing the patient should lie on the affected side to permit the free escape of discharge ; and a piece of absorbent wadding should be loosely placed in the meatus to soak up discharge and protect the ear from the injurious effects of cold and noise. From the time of the natural or artificial opening of the abscess, the Eustachian tube should be kept pervious-in the adult by the daily gentle use of Politzer's inflator, and in the child by the simple expedient of blowing air up the elosed nostril through a piece of india-rubber tubing. In a few days, when inflammation has somewhat abated, weak, warm, astringent solutions should be injected twice daily after cleansing with warm water. Solutions of sulphate of zinc or copper and the sulpho-carbolate of zinc are those which I have found most satisfactory. Vegetable infusions, on account of their liability to decomposition, should on no account be used. It is hardly necessary to say that after every wet application to the ear the meatus should be carefully dried by turning the head on its side, so as to allow the fluid to trickle out, and by using a piece of soft rag.

The line of treatment which I have rapidly sketched may at first sight appear elaborate and difficult of execution. But a moment's reflection will convince you that it is neither more nor less than the common-sense application of wellknown principles of surgery; and speaking from my own experience I have invariably found both patients and their friends willing co-operators when the objects of the treatment and the probable consequences of neglect have been fairly placed before them.

If I am asked what measure of success is likely to attend the adoption of this plan, I can only answer, from my own experience and that of numerous others, that the results are quite as good as could be expected from the rational treatment of an acute abscess in such a situation. Quite recently I have lad under my care two cases of acute suppuration of both middle ears in adults. In both cases perforation had occurred before I was consulted; and in both entire cessation of inflammatory action, perfect healing of the membranæ tympani, and complete restoration of hearing power were obtained within six weeks of the commencement of treat. ment.

If you admit, as I think everybody must, the truth of Sir William Wilde's observation, that "as long as discharge from the ear is present we can never know bow, when, or where it may terminate, or to what it may lead," if you think of the immediate and future dangers of tympanic suppuration, if you concede its evident disqualification for life assurance, and if you reflect on the restraining power of imperfect hearing in the keen and eager struggle for existence, rank, and wealth, - you will not fail to recognise the importance of accurate diagnosis and rational treatment, and you will find in the study of the diseases of the tympanum a field for the exercise of your best skill, care, and talents, a source of gratification to yourselves, and of inestimable benefit to your patients.

Dundee.

THE annual meeting of the governors of the Birkenhead Borough Hospital was held at the Music Hall, on Jan. 31st, the Mayor (Wm. Laird, Esq.) presiding. The report of the committee of management stated that the total number of patients relieved during the year 1880 was 7724 , of whorn 535 were in-patients. The total expenditure amounted to $\$ 2138$ 1ls. $3 d$, , being an increase of $£ 1301 s$. $9 d$. on the previous year. The total ordinary receipts for the year were $£ 23319 s .6 d$., an increase of $£ 4192 s$. $4 d$. on the previous year. The total receipts exceeded the disbursenients by $£ 19218 s .3 \mathrm{~d}$. The committee most gratefally acknowledge the handsome donation of $£ 250$ received from J. T. Hickells, Esq., of Noctorum; and a legacy of $£ 100$ (less duty) from the late J. Crowther, Esq., of Oxton. Owing to the yearly increasing number of in-patients the committee considered that the opening of the fourth ward had become a matter of pressing necessity. This, however, could not be done without adding $a$ wing to the main building and making some other slight alterations, at a probable cost of not less than $£ 600$. 